\title{
A review: Can robots reshape K-12 STEM education?
}

\author{
Mohammad Ehsanul Karim, Séverin Lemaignan, and Francesco Mondada \\ Laboratoire de Systèmes Robotiques \\ École Polytechnique Fédéral de Lausanne, EPFL \\ Email: \{ehsan.karim, severin.lemaignan, francesco.mondada\}@epfl.ch
}

\begin{abstract}
Can robots in classroom reshape K-12 STEM education, and foster new ways of learning? To sketch an answer, this article reviews, side-by-side, existing literature on robot-based learning activities featuring mathematics and physics (purposefully putting aside the well-studied field of "robots to teach robotics") and existing robot platforms and toolkits suited for classroom environment (in terms of cost, ease of use, orchestration load for the teacher, etc.). Our survey suggests that the use of robots in classroom has indeed moved from purely technology to education, to encompass new didactic fields. We however identified several shortcomings, in terms of robotic platforms and teaching environments, that contribute to the limited presence of robotics in existing curricula; the lack of specific teacher training being likely pivotal. Finally, we propose an educational framework merging the tangibility of robots with the advanced visibility of augmented reality.
\end{abstract}

\section{INTRODUCTION}

Robots have the potential to be the next effective addon to traditional education. The tangibility of robots and the excitement they bring into the classroom environment is considered conducive for learning. Such claims from anecdotal studies have inspired many schools to adopt robotics into their formal or informal curricula. However, the actual contribution of robots in science, technology, engineering and mathematics (STEM) education is not obvious; the fundamental question remains unanswered: Can robots in classroom reshape education and foster learning? In order to answer the question we survey existing literature to seek satisfactory evidence of robot-based learning. Specifically, our review focuses on the learning activities developed for teaching mathematics and physics in K-12 education.

The remainder of this section summarizes the organization of the paper. Section II provides an updated definition of educational robotics, and summarizes robot-based educational activities featuring mathematics and physics. Section III presents potential robotic platforms that are capable of being used in education and describes the system in terms of processor, battery life, sensors, cost, design and construction process. Finally, section IV constructively critiques the literature in an attempt to answer the titled question and proposes a potential future research direction.

\section{ROBOT-BASED LEARNING ACTIVITIES}

\section{A. Updated definition of educational robotics}

The term educational robotics is quite vague; the traditional definition predominantly involves developing technical knowledge by constructing and programming the robot [6],
TABLE I

SUMMARY OF THE TOPICS COVERED IN EDUCATIONAL ROBOTICS FEATURING MATHEMATICS AND PHYSICS.

\begin{tabular}{ll}
\hline Mathematics & Physics \\
\hline Geometric primitives [1], [2] & Distance, time, and velocity \\
Counting [3] & {$[11],[13]$} \\
Multiplication [4] & Constant speed, acceleration, \\
Decimals [5] & and deceleration [10] \\
Fractions and ratios [6]-[8] & Work and energy [14] \\
Coordinate system [2], [8] & Force, gravity, and friction [15] \\
Recognition of quantities [9] & Doppler effect [16] \\
Problems with operator [9] & Fundamentals of electricity [17] \\
$\begin{array}{l}\text { Graph construction and inter- } \\
\text { pretation [10], [11] }\end{array}$ & Weight scale and moment com- \\
Angles [11], [12] & putation [18] \\
\hline
\end{tabular}

[19], [20]. The definition is in-line with modern pedagogical theory of learning: (1) Papert's constructionism theory [1], (2) learning by design [21], (3) principles of active learning [22], and (4) social constructivism [23]. This definition focusing on the interactive learning activity is widely accepted, however embarrassingly narrow. Recent trend suggests that the learning domains have broadened, therefore the definition requires an update. Now-a-days, robots are being used to teach non-technical modules; for example, mathematics [24], [25], physics [11], language, and music [26]-[28]. Moreover, robots are socially assisting in the cognitive and intellectual development of children, as well [29]. The role of the robots in such activities is primarily as a tool for learning. However collaborative human-robot interactive learning and robotbased mentoring has also been demonstrated [11].

\section{B. Robotics and mathematics}

Robot-based learning of geometric primitives using the TURTLE/LOGO platform was first introduced in the 80s by Papert under the pedagogical framework of Constructionism [1]. Papert envisioned that student-robot interaction would improve knowledge and problem-solving skills because the introduction of robots in classroom would increase motivation and provide an experimental platform for practice [5], [30]. However, till-date not much significant achievements were made in this regard. Four decades later, Papert's results were reproduced using the iRobot Create in drawing two dimensional geometric primitives and advanced complex shapes; the study reported that as a by product students' sense of coordinate system was improved [2]. Few studies with middle schools [31], [32] reported that robots sig- 
nificantly helped in improving skills with fractions, ratios and coordinate estimation. Further studies emphasized on the importance of the involvement duration: longer robotic intervention effected content learning, while the shorter version effected motivation and attitude [8]. These findings were reinforced by studies which successfully demonstrated the learning of decimals [5] and ratios [6], [7]. A large scale study with 2nd, 3rd and 4th graders in Peru showed improvements in operation solving skills and recognition of quantities; and emphasized on the gender unbiased learning [9]. However, two major studies [33], [34] each involving one year of LEGO formal classroom training showed that only a certain group of 5 th grade students displayed improved performance in mathematics. However, these results cannot be definitive because positive engagement with robots involved students who had superior background in mathematics.

\section{Robotics and physics}

Existing literature reported several robot-based activities targeting the fundamentals of classical physics. Teachers reported that such activities improved motivation, engagement and attitude [30]. The students demonstrated creative thinking and practical learning of the concepts [16]. The topic of kinematics: the relationship between distance, time and velocity was collaboratively studied and discussed while constructing, programming and studying the motion of LEGObased robot by middle school students [11], [13], [35]. Kinetics-related activities targeting Newton's law of motion reported that the active manipulation of robot performance variables, like, forces, friction, weight, slope of the ramp, wheel diameter, etc. enhanced content knowledge [15]. Similar construction activities: solar powered car, rubber bandbased catapult, and wind-turbine helped teach standard forms of energy and energy conversion techniques [14]. Moreover, studies developed for teaching ratios while constructing robots with cogs and pulleys required theoretical and practical understanding of gear ratio and pulley mechanism. The research findings were promising: students displayed improvements in their written ability to explain science [7]. Furthermore, studies showed that robots helped students in the construction and interpretation of graphs [11]. Follow up studies displayed remarkable results strongly supporting for the use of robots compared to simulators [35]. Similar related studies showed that students can better represent and interpret position-time graphs relating to constant speed, acceleration and deceleration [10]. Please refer to Table I for a summary of the discussed activities.

\section{RoBotic Platforms fOr EdUCATION}

This section reports robots developed for education and summarizes the design specification in terms of processor, sensors, battery life and cost. Based on the design and construction complexity these robots are classified as: (1) complex LEGO-like brick-based robot assembly kit, (2) minimal mobile robot design kit, (3) robot manipulator design kit, (4) open-source robot design, (5) pre-assembled desktop robots and (6) miniaturized swarm robots.
Please note: The listed robots are mobile and manipulative in nature, and does not include humanoids, aerial, or other types; most importantly this list is not meant to be exhaustive. Furthermore, the information is comparatively presented to assist educators and researchers in the efficient selection of their desired robot platform.

\section{A. Brick-based robotic toolkits}

\begin{tabular}{|c|c|c|c|}
\hline Name & $\begin{array}{l}\text { Mindstorms } \\
\text { EV3 [36] }\end{array}$ & $\begin{array}{l}\text { VEX IQ } \\
\text { Super } \\
\text { Kit [37] }\end{array}$ & $\begin{array}{c}\text { ROBOTIX } \\
\text { TXT } \\
\text { Discovery } \\
\text { Kit [38] }\end{array}$ \\
\hline Company & LEGO & Vex Robotics & Fischertechnik \\
\hline Arm Processor & $\begin{array}{c}926 \mathrm{EJ}-\mathrm{S} \\
4 \mathrm{I} / \mathrm{O}\end{array}$ & $\begin{array}{c}\text { Cortex-M4 } \\
12 \mathrm{I} / \mathrm{O}\end{array}$ & $\begin{array}{c}\text { Cortex } \\
\mathrm{A} 8+\mathrm{M} 3\end{array}$ \\
\hline Programming & EV3 Software & $\begin{array}{c}\text { ROBOTC, } \\
\text { Modkit }\end{array}$ & $\begin{array}{c}\text { RoboPro } \\
\mathrm{C} / \mathrm{C}++\end{array}$ \\
\hline $\begin{array}{l}\text { Visual } \\
\text { Programming }\end{array}$ & yes & yes & yes \\
\hline Sensors & $\begin{array}{l}\text { Speaker } \\
\text { Touch } \\
\text { Color } \\
\text { Distance } \\
\text { Bump } \\
3 \text { Motors } \\
\text { Encoders }\end{array}$ & $\begin{array}{c}\text { Speaker } \\
\text { Touch } \\
\text { Color } \\
\text { Distance } \\
\text { Bump } \\
4 \text { Motors } \\
\text { Joystick } \\
\text { Gyro } \\
\text { Radio }\end{array}$ & $\begin{array}{c}\text { Speaker } \\
\text { Touch } \\
\text { Color } \\
2 \text { LEDs } \\
\text { Camera } \\
\text { Switch } \\
2 \text { Motors } \\
\text { Encoders }\end{array}$ \\
\hline Design Demo & 5 & 1 & 14 \\
\hline Connection & $\begin{array}{c}\text { Bluetooth + } \\
\text { USB + } \\
\text { Radio }\end{array}$ & $\begin{array}{c}\text { USB + } \\
\text { Blue-tooth }\end{array}$ & $\begin{array}{c}\text { Blue-tooth + } \\
\text { Wifi + } \\
\text { Radio + USB }\end{array}$ \\
\hline Add-ons & $\begin{array}{c}\text { EV3 Gyro } \\
\text { (add: } \$ 30.00)\end{array}$ & $\begin{array}{c}\text { Omni- }^{-} \\
\text {directional } \\
\text { wheels, Tank } \\
\text { chain tread } \\
\text { (add: } \$ 100.00)\end{array}$ & $\begin{array}{c}\text { Power Set } \\
\text { RoboPro } \\
\text { (add: } \$ 115.00)\end{array}$ \\
\hline Cost & $\$ 350.00$ & $\$ 330.00-360.00$ & $\$ 550.00$ \\
\hline
\end{tabular}

TABLE II

BRICK-BASED (LEGO-LIKE) ROBOT ASSEMBLY KITS.

The robot design kit used in most of the educational activities was primarily the LEGO Mindstorms NXT 2.0. The kit consists of LEGO bricks, motors, gears, sensors (ultrasonic, sound, touch, and color) which can be programmed using the NXT multipurpose controller to design multiple custom shaped robots. The robot-kit documentation provides instruction for constructing four unique robots which includes wheeled, manipulator-like robots or both [39]. Table II summaries some of the available commercial platforms. Despite the wide usage of LEGO-like kits in STEM education, the required formal involvement in the classroom environment is largely missing. Most activities are shorttermed and developed informally through extra-curricular activities [40]. The reason is primarily associated to the time consuming unintuitive overwhelming design process which requires excellent inventory and project management skills. As a direct consequence, teachers control over the classroom is reduced, which worsens due to the absence of formal structured curricula linking traditional and robotbased education. Thus the role of the teacher as facilitator, 
educator, or guide is minimized. Most importantly, given the constrained budget in primary and secondary schools these kits are not always affordable.

\section{B. Modular robotic kits}

To get around the issue of complex construction process, there exists commercial robotic kits which provide minimal design space compared to LEGO-like kits, thereby minimizing the assembly time. Table III and IV provide a compact overview of some of these commercial kits; in particular we focus on wheeled and armed platforms. The reasonable cost and the limited design space provide a convenient platform for classroom. Moreover it provides the students with construction satisfaction while shortening the assembly time, and teachers with enough time to develop and introduce curricula centric activities. Such combination provides space for improved student-teacher collaboration. Table V, in contrast, lists similar open-source robot designs; these robots are not commercially available but can be made using COTS components. The design process is quite involved; it requires soldering, wiring, PCB design and programming. Traditionally, the design is limited to building and programming a differential drive line follower robot with a unique set of sensors. Under proper guidance, the activity could allow students to develop knowledge of electricity, magnetism, sensors, and mechanics. Most importantly, the price is quite reasonable and design space is expandable.

\begin{tabular}{|l|c|c|c|c|}
\hline Name & $\begin{array}{c}\text { Servo- } \\
\text { botics } \\
\text { RA-02 } \\
{[48]}\end{array}$ & $\begin{array}{c}\text { Lynx } \\
\text { AL5x } \\
{[49]}\end{array}$ & $\begin{array}{c}\text { Robotic } \\
\text { Arm } \\
\text { Edge } \\
{[50]}\end{array}$ & $\begin{array}{c}\text { Arxx } \\
\text { Robot } \\
\text { Mini } \\
{[51]}\end{array}$ \\
\hline $\begin{array}{l}\text { Degrees of } \\
\text { Freedom }\end{array}$ & 5 & 5 & 5 & 6 \\
\hline Motor & servo & servo & servo & servo \\
\hline Payload (gr.) & very small & $100-283$ & 100 & $\mathrm{x}$ \\
\hline Height (cm) & 40 & 40 & 38 & 32 \\
\hline Reach (cm) & 31 & $15-20$ & 30 & 26 \\
\hline Programmable & yes & yes & yes & C/C++ \\
\hline Connection & Serial & USB & USB & USB \\
\hline Other & & & & 4 LEDs \\
\hline Cost & $\$ 250.00$ & $\$ 360.00$ & $\$ 66.00$ & $\$ 150.00$ \\
\hline
\end{tabular}

TABLE IV

COMMERCIAL LOW COST PROGRAMMABLE ROBOT MANIPULATORS.

\section{Pre-assembled robots}

Pre-assembled robots are compact in size and can be installed indoor to work alongside desktop computers. Most of these robots are initially developed at universities for teaching engineering courses and later have been commercialized; for example, the E-puck [59] has been successfully adopted at EPFL and several other robotics laboratories allaround the world. Some of the robots in these category are listed in Table VI and VII. The final class of indoor robots are the miniaturized version of these pre-assembled platforms. Individually, these robots have minimal sensing capabilities, however as a group they are extremely capable. The hardware design is open-source, and the required COTS

\begin{tabular}{|c|c|c|c|}
\hline Name & Hemission [60] & $\begin{array}{c}\text { K-Junior } \\
\text { V1 [60] }\end{array}$ & $\begin{array}{c}\text { K-Junior } \\
\text { V2 [60] }\end{array}$ \\
\hline PIC Processor & $16 \mathrm{~F} 877$ & $16 \mathrm{~F} 877$ & $18 \mathrm{~F} 66 \mathrm{~K} 22$ \\
\hline Encoders & $\mathrm{x}$ & $\mathrm{x}$ & $\mathrm{x}$ \\
\hline DC Motor & 2 & 2 & 2 \\
\hline Buzzer & yes & yes & yes \\
\hline Switches & 4 & 3 & 3 \\
\hline Diameter (cm) & 12 & 12.5 & 12.5 \\
\hline Battery Life (hours) & 2 & 4 & 4 \\
\hline Obstacle detectors & 6 & $\mathrm{x}$ & $\mathrm{x}$ \\
\hline IR ground sensor & 2 & 4 & 4 \\
\hline Programmable LEDs & 4 & 3 & 5 \\
\hline $\begin{array}{c}\text { IR proximity and } \\
\text { ambient light sensors }\end{array}$ & 8 & 6 & 6 \\
\hline Visual Programming & yes & yes & yes \\
\hline Cost & $\$ 250.00$ & $\$ 900.00$ & $\$ 800.00$ \\
\hline
\end{tabular}

TABLE VI

EDUCATIONAL ROBOTS FROM THE K-TEAM.

parts are easily obtainable. Table VIII lists some of these robots. These robots are particularly designed for swarmrelated research; however their size and the production cost make them well-suited for K-12 STEM education where each student would have his/her own robot and will collaborate with peers and teachers through the learning activities.

Two noteworthy designs for academic research and education: The EPFL Thymio [61] and the Infante [72]. EPFL Thymio [61] is one of the most successful commercial fullassembled robot; the platform is re-programmable, rich in sensors and actuators, rechargeable, supports Lego modularity and appreciated by many students. Meanwhile, the Infante robot displayed promising results; studies have reported successful implementation of activities pertaining to mathematical reasoning, geography and recycling [72]. Theoretically, most of the aforestated robots could have replaced the LEGO platform for most of the learning activities mentioned in section II; nevertheless educators use LEGO.

TABLE IX

AR-BASED EDUCATION FEATURING MATHEMATICS AND PHYSICS.

\begin{tabular}{ll}
\hline Mathematics & Physics \\
\hline $\begin{array}{l}\text { Construct3D: Geometry [73] } \\
\text { CyberChase Shape Quest: Ge- } \\
\text { ometry, spatial reasoning and } \\
\text { problem solving [74] }\end{array}$ & $\begin{array}{l}\text { Physics Playground: Kinetics } \\
\text { CyberMath: Differential geom- }\end{array}$ \\
$\begin{array}{l}\text { kinematics in 1D [7 [78] } \\
\text { etry [75] }\end{array}$ & $\begin{array}{l}\text { Maxwell World: Fundamentals } \\
\text { of electromagnetism [78] }\end{array}$ \\
Alien Contact! Basic mathe- & Graph interpretation [77] \\
matics, and problem solving & Elastic collisions [79], [80] \\
[76] & Doppler effect [81] \\
& Principles of aircraft flight [81] \\
& Work and energy [14] \\
& Electricity and magnetism [82] \\
& ARex: Light experiment with \\
& prism [83] \\
& Fundamentals of lens [84] \\
& Weight scale and moment com- \\
& putation [18] \\
\hline
\end{tabular}




\begin{tabular}{|c|c|c|c|c|c|c|c|}
\hline Name & Mark III [41] & $\begin{array}{l}\text { Polulo } \\
\text { 3-Pi [42] }\end{array}$ & $\begin{array}{c}\text { Pololu Zumo } \\
\text { 32U4 Robot [43] }\end{array}$ & $\begin{array}{c}\text { Arduino } \\
\text { Starter Kit [44] }\end{array}$ & Boe-bot [45] & Qfix [46] & ARobot [47] \\
\hline Processor & PICF877 & ATmega328 & ATmega32U4 & ATmega328 & Basic Stamp II & ATmega128 & $\mathrm{x}$ \\
\hline Programming & $\mathrm{C} / \mathrm{C}++$ & $\mathrm{C} / \mathrm{C}++$ & $\mathrm{C} / \mathrm{C}++$ & $\begin{array}{c}\text { Visual } \\
\text { Programming: } \\
\text { minibloq, } \\
\text { ArduBlock }\end{array}$ & PBASIC & $\begin{array}{c}\mathrm{C} / \mathrm{C}++, \\
\text { visually } \\
\text { program- } \\
\text { mable }\end{array}$ & $\mathrm{x}$ \\
\hline Diameter $(\mathrm{cm})$ & $\mathrm{X}$ & 10 & 10 & $\mathrm{x}$ & $\mathrm{x}$ & $\mathrm{x}$ & $\mathrm{x}$ \\
\hline Drive System & differential & $\begin{array}{l}\text { tank-style } \\
\text { differential }\end{array}$ & $\begin{array}{l}\text { tank-style } \\
\text { differential }\end{array}$ & differential & differential & $\begin{array}{c}\text { omni- } \\
\text { directional }\end{array}$ & $\begin{array}{l}\text { front wheel drive, } \\
\text { rear wheel steer }\end{array}$ \\
\hline Motors & $\begin{array}{l}2 \text { torque } \\
\text { servo }\end{array}$ & $\begin{array}{l}2 \text { micro-metal } \\
\text { gear }\end{array}$ & $\begin{array}{l}2 \text { micro-metal } \\
\text { gear }\end{array}$ & 1 servo, $3 \mathrm{DC}$ & $\begin{array}{l}2 \text { continued } \\
\text { rotation servo }\end{array}$ & 3 gear & rc servo, DC gear \\
\hline Encoder & yes & $\mathrm{x}$ & yes & $\mathrm{x}$ & $\mathrm{x}$ & $\mathrm{x}$ & $\mathrm{X}$ \\
\hline Proximity & 2 & $\mathrm{x}$ & 4 & 1 ultrasound & $\begin{array}{c}3 \\
\text { (ultrasound + IR) }\end{array}$ & 2 & $\mathrm{x}$ \\
\hline Ground Sensor & 3 & 5 & 5 & $\mathrm{x}$ & & 1 & $\mathrm{x}$ \\
\hline Push-botton & $\mathrm{X}$ & 3 & 3 & 10 & 2 & 2 & 2 \\
\hline LEDs & $\mathrm{x}$ & $\mathrm{x}$ & yes & 27 & $\mathrm{x}$ & 2 & 2 \\
\hline Sound & $\mathrm{X}$ & buzzer & buzzer & $\mathrm{x}$ & $\mathrm{X}$ & $\mathrm{X}$ & buzzer \\
\hline LCD & $\mathrm{X}$ & 82 characters & 82 characters & $\mathrm{x}$ & $\mathrm{x}$ & $\mathrm{x}$ & $\mathrm{x}$ \\
\hline Connection & Serial & $\mathrm{X}$ & USB & USB & USB, Serial & USB & $\mathrm{X}$ \\
\hline Other & $\mathrm{x}$ & $\mathrm{x}$ & $\begin{array}{l}\text { gyro, compass, } \\
\text { accelerometer }\end{array}$ & $\begin{array}{c}\text { tilt, } 6 \text { lights, } \\
\text { and temperature } \\
\text { sensor, } 1 \text { IMU } \\
\text { with altitude }\end{array}$ & $\begin{array}{l}\text { light sensor, } \\
2 \text { bumpers }\end{array}$ & $\mathrm{x}$ & $\begin{array}{c}2 \text { whiskers, PIR } \\
\text { motion, light, } \\
\text { temperature } \\
\text { sensors }\end{array}$ \\
\hline Cost & $\$ 92.00$ & $\$ 100.00$ & $\$ 100.00$ & $\$ 156.00$ & $\$ 160.00$ & $\$ 310.00$ & $\$ 395.00$ \\
\hline
\end{tabular}

TABLE III

COMMERCIAL LOW COST MINIMAL MOBILE ROBOT DESIGN KITS.

\begin{tabular}{|c|c|c|c|c|c|c|c|}
\hline Name & MIT SEG [52] & $\begin{array}{c}\text { Harvard } \\
\text { Kilobot [53] }\end{array}$ & $\begin{array}{c}\text { Rice } \\
\text { R-one [54] }\end{array}$ & Infante [55] & $\begin{array}{c}\text { Miniskybot } \\
1.0[56]\end{array}$ & Pi Swarm [57] & $\begin{array}{l}\text { Evolution } \\
\text { ER1 [58] }\end{array}$ \\
\hline Battery (hours) & $\mathrm{x}$ & $3-24$ & 4 & $\mathrm{x}$ & $\mathrm{x}$ & 2 & $\mathrm{x}$ \\
\hline Processor & $\begin{array}{l}\text { Arduino Pro } \\
\text { Mini }\end{array}$ & Atmega328 & $\begin{array}{c}\text { LM3S8962 } \\
\text { Stellaris Processor }\end{array}$ & PIC16F88 & PIC16F876A & ARM Cortex-M3 & Laptop \\
\hline Programming & $\begin{array}{c}\text { ArduBlock } \\
\text { Graphical } \\
\text { Programming }\end{array}$ & $\mathrm{C} / \mathrm{C}++$ & $\begin{array}{l}\text { C/C++ } \\
\text { Python }\end{array}$ & $\begin{array}{c}\text { Blocky } \\
\text { Graphical } \\
\text { Programming }\end{array}$ & $\mathrm{C} / \mathrm{C}++$ & $\mathrm{C} / \mathrm{C}++$ & $\begin{array}{l}\mathrm{C} / \mathrm{C}++, \\
\text { Python }\end{array}$ \\
\hline Dimension $(\mathrm{cm})$ & $\mathrm{x}$ & diameter: 3 & diameter: 11 & $\mathrm{x}$ & $\mathrm{x}$ & diameter: 9.5 & $\mathrm{x}$ \\
\hline Drive System & differential & differential & differential & differential & differential & differential & differential \\
\hline Motors & 2 servo & 2 vibrator & 2 geared & 2 rc servo & 2 servo & 2 micro-metal gear & 2 stepper \\
\hline Encoder & $\mathrm{x}$ & $\mathrm{x}$ & yes & $\mathrm{x}$ & $\mathrm{x}$ & $\mathrm{x}$ & yes \\
\hline Proximity & $\mathrm{x}$ & yes & 8 & $\mathrm{x}$ & 2 ultrasound & 8 & 3 \\
\hline Ground Sensor & 1 & $\mathrm{x}$ & $\mathrm{x}$ & 4 & $\mathrm{x}$ & 5 & $\mathrm{x}$ \\
\hline Push-botton & $\mathrm{x}$ & $\mathrm{x}$ & 3 & $\mathrm{x}$ & $\mathrm{x}$ & 3 & $\mathrm{x}$ \\
\hline LEDs & $\mathrm{x}$ & $\mathrm{x}$ & 15 & $\mathrm{x}$ & $\mathrm{x}$ & 11 & $\mathrm{x}$ \\
\hline Sound & $\mathrm{x}$ & $\mathrm{x}$ & speaker & $\mathrm{x}$ & $\mathrm{x}$ & buzzer & $\mathrm{x}$ \\
\hline Connection & Blue-tooth & $\begin{array}{c}\text { IR-based } \\
\text { Kilobot } \\
\text { controller }\end{array}$ & Radio (2.4 Ghz) & Bluetooth & Serial & $\begin{array}{l}\text { USB, RF } \\
\text { Transceiver }\end{array}$ & Serial \\
\hline Other & $\mathrm{x}$ & $\begin{array}{l}\text { ambient light, } \\
\text { inter-robot } \\
\text { communication }\end{array}$ & $\begin{array}{c}8 \text { bumpers, } 4 \\
\text { ambient lights, } \\
\text { IR beacon global } \\
\text { localization, gyro, } \\
\text { compass, } \\
\text { accelerometer, } \\
\text { SC card, planner } \\
\text { gripper }\end{array}$ & $\begin{array}{c}\text { Raspberry-Pi } \\
\text { based } \\
\text { multi-robot } \\
\text { controller }\end{array}$ & $\mathrm{x}$ & $\begin{array}{l}\text { 3D gyro, compass, } \\
\text { accelerometer, } \\
\text { temperature, } \\
\text { light, ultrasonic } \\
\text { range }\end{array}$ & camera \\
\hline Cost & $\$ 20.27$ & $\$ 43.00$ & $\$ 300.00$ & $\begin{array}{c}\$ 110.00 \\
(5 \text { robots })\end{array}$ & $\$ 65.00$ & $\$ 245.00$ & $\$ 900.00$ \\
\hline
\end{tabular}

TABLE V

OPEN-SOURCE LOW COST MOBILE PLATFORMS DESIGNED FROM COMMERCIAL OFF-THE-SHELF COMPONENTS. 


\begin{tabular}{|c|c|c|c|c|c|c|}
\hline Name & Thymio [61] & $\begin{array}{c}\text { iRobot } \\
\text { Create [62] }\end{array}$ & $\begin{array}{l}\text { Wow-Wee } \\
\text { Rovio [63] }\end{array}$ & $\begin{array}{c}\text { Surveyor } \\
\text { SRV-1 [64] }\end{array}$ & E-puck [59] & AmigoBot [65] \\
\hline Battery (Hours) & 2 & 1.5 & 2 & 4 & 3 & 2 \\
\hline Processor & PIC24FJ128 & ATmega 168 & $\begin{array}{c}\text { Marvell } \\
\text { PXA270M ARM }\end{array}$ & $\begin{array}{l}\text { Blackfin } \\
\text { BF537 }\end{array}$ & dsPIC & Hitachi H8 \\
\hline Programming & $\begin{array}{l}\text { ASEBA, Visual } \\
\text { Programming }\end{array}$ & $\mathrm{C} / \mathrm{C}++$ & $\begin{array}{l}\text { C/C++ } \\
\text { Python }\end{array}$ & $\begin{array}{l}\mathrm{C} / \mathrm{C}++ \\
\text { Python }\end{array}$ & $\mathrm{C} / \mathrm{C}++$ & $\mathrm{C} / \mathrm{C}++$ \\
\hline Dimension $(\mathrm{cm})$ & $10 \times 10$ & diameter: 13 & diameter: 11 & $12 \times 10.5$ & diameter: 7 & $32 \times 28$ \\
\hline Drive System & differential & differential & omni-direction & $\begin{array}{c}\text { differential } \\
\text { tank-style }\end{array}$ & differential & differential \\
\hline Motors & 2 DC gear & 2 vibrator & 2 wheel, 1 camera & 4 DC gear & $\begin{array}{l}2 \text { stepper } \\
\text { geared }\end{array}$ & $2 \mathrm{DC}$ \\
\hline Encoder & not directly & yes & yes & $\mathrm{x}$ & yes & yes \\
\hline Proximity & 7 & 1 & yes & 2 & 8 & 8 ultrasound \\
\hline Ground Sensor & 2 & 4 cliff sensors & $\mathrm{x}$ & 4 & $\mathrm{x}$ & $\mathrm{x}$ \\
\hline Push-botton & 5 touch sensors & $\mathrm{X}$ & 3 & $\mathrm{X}$ & $\mathrm{x}$ & $\mathrm{x}$ \\
\hline LEDs & 39 & 3 & yes & $\mathrm{x}$ & 10 & $\mathrm{x}$ \\
\hline Sound & $\begin{array}{c}\text { speaker, } \\
\text { microphone }\end{array}$ & speaker & $\begin{array}{c}\text { speaker, } \\
\text { microphone }\end{array}$ & & $\begin{array}{c}\text { speaker, } 3 \text { omni- } \\
\text { directional microphones }\end{array}$ & buzzer \\
\hline Connection & USB & Serial & Wireless & Wi-Fi & Bluetooth, Serial & Serial, Wireless \\
\hline Other Specs. & $\begin{array}{l}\text { IR communication, } \\
\text { 3D accelerometer, } \\
\text { temperature } \\
\text { sensors }\end{array}$ & $\begin{array}{l}3 \text { bumpers, } \\
\text { IR communication, } \\
\text { attachable camera }\end{array}$ & $\begin{array}{l}\text { VGA camera, } \\
\text { indoor localization } \\
\text { using True Beacon } \\
\text { NorthStar }\end{array}$ & $\begin{array}{l}1.3 \mathrm{MP} \\
\text { Camera }\end{array}$ & $\begin{array}{l}\text { IR communication } \\
\text { VGA camera } \\
\text { 3D accelerometer, } \\
\text { ambient light sensors }\end{array}$ & $\mathrm{x}$ \\
\hline Cost & $\$ 105.00$ & $\$ 200.00$ & $\$ 270.00$ & $\$ 480.00$ & $\$ 850.00$ & $\$ 1800.00$ \\
\hline
\end{tabular}

TABLE VII

LOW-COST FULLY-ASSEMBLED COMMERCIAL MOBILE ROBOTS.

\begin{tabular}{|c|c|c|c|c|c|c|}
\hline Name & CotsBots [66] & Robomote [67] & Alice [68] & TERMES [69] & MICAbot [70] & Kobot [71] \\
\hline Battery (Hours) & 1 & 3.5 & 10 & $\mathrm{X}$ & 2.5 & $7-10$ \\
\hline Processor & ATmega128L & AT90S8535L & PIC16F84 & ATmega128L & ATmega103L & PIC16F877A \\
\hline Size (in $\mathrm{cm}$ ) & $13 \times 6.5$ & $3.81 \times 2.23$ & $2.1 \times 2.1$ & $17 \times 11$ & $8.6 \times 6.1$ & diameter: 12 \\
\hline Motors & 2 servo & $2 \mathrm{DC}$ & 2 swatch, $1 \mathrm{DC}$ & 2 micro-metal gear & 2 sub-micro servo & $\begin{array}{l}2 \text { high torque } \\
\text { DC gear-head }\end{array}$ \\
\hline Drive System & $\begin{array}{c}4 \text { wheel } \\
\text { Ackermann }\end{array}$ & differential & $\begin{array}{c}\text { differential } \\
\text { all-terrrain } \\
\text { caterpillar thread }\end{array}$ & $\begin{array}{l}4 \text { carved wheg } \\
\text { differential }\end{array}$ & differential & differential \\
\hline Encoder & $\mathrm{X}$ & yes & $\mathrm{X}$ & $\mathrm{X}$ & yes & $\mathrm{X}$ \\
\hline Proximity & $\mathrm{X}$ & 5 & 4 & $\mathrm{X}$ & $\mathrm{X}$ & 8 \\
\hline Bumper & 4 & 4 & $\mathrm{X}$ & $\mathrm{X}$ & $\mathrm{X}$ & $\mathrm{x}$ \\
\hline $\begin{array}{l}\text { Inter-robot } \\
\text { comm }\end{array}$ & yes & yes & yes & $\mathrm{x}$ & yes & $\mathrm{x}$ \\
\hline Programming & TinyOS & $\mathrm{C} / \mathrm{C}++$ & $\mathrm{C} / \mathrm{C}++$ & $\mathrm{C} / \mathrm{C}++$ & TinyOS & $\mathrm{x}$ \\
\hline Sound & $\begin{array}{l}\text { microphone, } \\
\text { buzzer }\end{array}$ & $\mathrm{x}$ & $\mathrm{x}$ & $\mathrm{x}$ & microphone & $\mathrm{x}$ \\
\hline Connectivity & RF Transceiver & RF Transceiver & IR, Radio & Blue-tooth & RF Transceiver & Wireless \\
\hline Other Specs. & $\begin{array}{l}\text { 2D compass, } \\
\text { accelerometer, } \\
\text { radio-based } \\
\text { distance, light, } \\
\text { temperature } \\
\text { sensors }\end{array}$ & $\begin{array}{l}\text { solar-cell } \\
\text { compass }\end{array}$ & $\begin{array}{l}\text { linear camera } \\
2 \mathrm{D} \text { gripper }\end{array}$ & $\begin{array}{c}6 \text { ground sensors, } \\
\text { tilt sensors, } \\
2 \text { DOF tactile } \\
\text { claw-like gripper } \\
3 \text { LEDs }\end{array}$ & $\begin{array}{l}\text { 2D compass, } \\
\text { accelerometer, } \\
\text { radio-based } \\
\text { distance, light, } \\
\text { temperature } \\
\text { sensors }\end{array}$ & $\begin{array}{l}\text { camera } \\
\text { compass }\end{array}$ \\
\hline Cost & $\$ 200.00$ & $\$ 150.00$ & $\$ 50.00$ & $\$ 100.00$ & $\$ 350.00$ & $\mathrm{x}$ \\
\hline
\end{tabular}

TABLE VIII

OPEN-SOURCE MiNiATURIZED SWARM RoBOTS. 


\section{Discussion AND CONCLUSION}

The use of robots to learn programming and robotics is well established [40], [85]. Our survey indicates that the field has evolved from conventional robotics education to non-technical learning activities as well - a positive development. Majority of the aforestated studies advocate that robots play a positive role in the learning of educational activities, develop creative thinking, and improve problemsolving skills. Moreover the interaction with robots increases motivation, engagement and attitude towards education. Admittedly, with the simplification of robot design and assembly process; the inclusion of intuitive visual dragand-drop programming and the gradually reducing cost of educational robot platforms, we are experiencing the advent of a new era in educational technology. However, further advancement would require identification of potential limitations and subsequent rational adaptation.

Based on our study, we identified two important issues which require our attention: (1) standardization of evaluation techniques which are used to quantify robot-based learning: merging statistical analysis, surveys and interviews and (2) development of tailored robot-based pedagogical modules assisting traditional K-12 curricula and associated teacher training programs [86].

The evaluation technique used to quantify the learning from the robot based activities, in most of the studies, is either quantitative or qualitative; ideally it is important to include both. Moreover, careful attention should be devoted in the design of the experiments in terms of sample size and sampling technique. The robust and standard approach is to use random sampling with appropriate sample size [87]; however, many of the studies do not comply. In addition, extreme caution should be exercised in planning the learning activities for the experimental and control group [87]. Furthermore, the quantification of learning must be based on multiple pre, post and retention tests to avoid outliers; the present standard of one pre and post test with substandard questions is not sufficient.

Teacher-student interaction is pivotal; the robot-based activities should be strongly linked with traditional curricula so robots could assist the teachers in day-to-day teaching activities. Because in some studies the students does not perceive or cannot link the learning goals and the robotbased activities. Such decoupling voids the link between use of robot and the associated increase in motivation to learn. This is a problematic issue because increase in motivation has been the crucial sales pitch in educational robotics.

Teachers are the most vital organ in the educational framework; they need to feel comfortable with robots. Such comfortability can only be achieved through proper training and active/pro-active involvement. Many of the teachers are hesitant in dealing with robotics, such behavior does not reflect their unwillingness to learn new concepts, sometimes it can be associated to the lack of standard curricula, and the affiliated long term benefit [86]. These limitations can be minimized by developing standardized and proven stable long-term curricula and related teacher training programs.

Technology is not replacing teachers, rather it is utilized to assist the teachers and the students to create a conducive epistemologically plural learning environment. To reach such milestone, standard curricula needs to be appropriately researched and subsequently adjusted so that it can aptly host the introduction of robot-based activities. Moreover teachers need to be properly trained, and the selection of the technology should be appropriate. For example not all robots can be used for the learning of verbal languages. Furthermore, educational framework should explicitly encourage both individual and collaborative learning.

Our experiences with the Thymio in schools [61], [88], [89] have helped us to realize that the student-robot interactivity and subsequent learning experiences can be further enhanced through the integration of a robot state or affordance visualizer; robots need to be more transparent in their mechanics. Such realization have made us interested in pursuing a new research direction by combining robotics and augmented reality (AR). Augmented reality (AR) is an interactive visualization technique (using cell-phones, tablets, head-mounted visual gears, etc) combining the real and the virtual world through accurate computer vision-based techniques [73], [77]. Our actual intent is to merge the tangibility of robots with the advanced visualization capabilities of AR. The purpose of AR is to display, in real-time, the invisible states and affordances of the robot. We strongly believe that the inclusion of AR would help the students in better visualization of invisible and abstract concepts, like: vectors, forces, gravity, geometry, electromagnetics, etc; and thereby further assist the K-12 mathematics and physics curricula. Based on our short survey of related AR-based educational activity, as summarized in table IX, it is found that AR technology is pedagogically effective for teaching mathematics and physics as it enhances user visualization capabilities, moreover the interactivity increases student motivation and improves learning [90], [91].

\section{ACKNOWLEDGMENT}

The authors would like to thank Fanny Riedo, Marcelo Elias De Oliveira, and Stefan Witwicki for their helpful suggestions and comments. This study was supported by National Centre of Competence in Research (NCCR) Robotics, Switzerland and is part of the project NCCR Transversal Educational Activity (TEA).

\section{REFERENCES}

[1] Seymour Papert. Mindstorms: Children, computers, and powerful ideas. Basic Books, Inc., 1980.

[2] Erin Walker and Winslow Burleson. User-centered design of a teachable robot. In Intelligent Tutoring Systems, pages 243-249. Springer, 2012. 
[3] Amanda Sullivan, Elizabeth R Kazakoff, and Marina Umashi Bers. The wheels on the bot go round and round: Robotics curriculum in pre-kindergarten. Journal of Information Technology Education, 12, 2013.

[4] Chun-Wang Wei, I Hung, Ling Lee, Nian-Shing Chen, et al. A joyful classroom learning system with robot learning companion for children to learn mathematics multiplication. Turkish Online Journal of Educational Technology-TOJET, 10(2):11-23, 2011.

[5] MD Portsmore and C Rogers. Bringing engineering to elementary school. Journal of STEM education, 5, 2004.

[6] Bradley S Barker and John Ansorge. Robotics as means to increase achievement scores in an informal learning environment. Journal of Research on Technology in Education, 39(3):229-243, 2007.

[7] Stephen John Norton. Using lego construction to develop ratio understanding. Mathematics education for the third millennium: Towards 2010, pages 414-421, 2004.

[8] Gwen Nugent, Bradley Barker, Neal Grandgenett, and Viacheslav I Adamchuk. Impact of robotics and geospatial technology interventions on youth stem learning and attitudes. Journal of Research on Technology in Education, 42(4):391-408, 2010.

[9] IM Iturrizaga and A Falbel. Study of educational impact of the lego dacta materials - infoescuela-med. Final Report, retrieved June, 30:2006, 2000.

[10] Dimitris Alimisis and George Boulougaris. Robotics in physics education: Fostering graphing abilities in kinematics.

[11] Ruben Mitnik, Miguel Nussbaum, and Alvaro Soto. An autonomous educational mobile robot mediator. Autonomous Robots, 25(4):367$382,2008$.

[12] Nicklas Sørlie Utgaard. Investigating the effect of a robotic presence compared to a virtual robot in teaching angles and turn measurements to children. 2014.

[13] Tassos A Mikropoulos and Ioanna Bellou. Educational robotics as mindtools. Themes in Science and Technology Education, 6(1):pp-5, 2013.

[14] Can Saygin, Timothy Yuen, Heather Shipley, H Wan, and David Akopian. Design, development, and implementation of educational robotics activities for k-12 students. In Proceedings of 2012 American Society for Engineering Education Annual Conference \& Exposition, 2012.

[15] Douglas C Williams, Yuxin Ma, Louise Prejean, Mary Jane Ford, and Guolin Lai. Acquisition of physics content knowledge and scientific inquiry skills in a robotics summer camp. Journal of Research on Technology in Education, 40(2):201-216, 2007.

[16] Jonathan Ashdown and David Doria. A robotics based design activity to teach the doppler effect. In Integrated STEM Education Conference (ISEC), 2012 IEEE 2nd, pages 1-3. IEEE, 2012.

[17] Heath Tims, Krystal Corbett, David Hall, Galen Turner, and Davis Harbour. Work in progress application of the boe-bot in teaching k12 electricity fundamentals. In Frontiers in Education Conference (FIE), 2011, pages S2D-1. IEEE, 2011.

[18] Aseba and Thymio. Moment computation on the classical weight scale. https://aseba.wikidot.com/en:thymioscale. Accessed March 19, 2015.

[19] Tucker Balch, Jay Summet, Doug Blank, Deepak Kumar, Mark Guzdial, Keith O'Hara, Daniel Walker, Monica Sweat, Gaurav Gupta, Stewart Tansley, et al. Designing personal robots for education: Hardware, software, and curriculum. IEEE Pervasive Computing, 7(2):5-9, 2008.

[20] Thomas Hsiu, Steve Richards, Ajinkya Bhave, Andres PerezBergquist, and Illah Nourbakhsh. Designing a low-cost, expressive educational robot. In Intelligent Robots and Systems, 2003.(IROS 2003). Proceedings. 2003 IEEE/RSJ International Conference on, volume 3, pages 2404-2409. IEEE, 2003.

[21] Rachel Goldman, Amy Eguchi, and Elizabeth Sklar. Using educational robotics to engage inner-city students with technology. In Proceedings of the 6th international conference on Learning sciences, pages 214221. International Society of the Learning Sciences, 2004.

[22] Merrill Harmin and Melanie Toth. Inspiring active learning: A complete handbook for today's teachers. ASCD, 2006.

[23] Lev S Vygotsky. Mind in society: The development of higher psychological processes. Harvard university press, 1980.

[24] Tertl Studos LLC. Robotmath: An innovative, hands-on approach to the foundations of algebra. http://tertl.com/robotmath/. Accessed March 19, 2015.
[25] Kate Highfield, Joanne Mulligan, John Hedberg, et al. Early mathematics learning through exploration with programmable toys. Paper submitted to PME, 31(2), 2008.

[26] Jeonghye Han and Dongho Kim. r-learning services for elementary school students with a teaching assistant robot. In Human-Robot Interaction (HRI), 2009 4th ACM/IEEE International Conference on, pages 255-256. IEEE, 2009.

[27] Takayuki Kanda, Takayuki Hirano, Daniel Eaton, and Hiroshi Ishiguro. Interactive robots as social partners and peer tutors for children: A field trial. Human-computer interaction, 19(1):61-84, 2004.

[28] Jeong-Hye Han, Dong-Ho Kim, and Jong-Won Kim. Physical learning activities with a teaching assistant robot in elementary school music class. In INC, IMS and IDC, 2009. NCM'09. Fifth International Joint Conference on, pages 1406-1410. IEEE, 2009.

[29] Maja J Mataric. Socially assistive robotics. Science, 3(6):233-242, 1999.

[30] Mike Robinson. Robotics-driven activities: Can they improve middle school science learning? Bulletin of Science, Technology \& Society, 25(1):73-84, 2005.

[31] Gwen Nugent, Brad Barker, and Neal Grandgenett. The effect of 4-h robotics and geospatial technologies on science, technology, engineering, and mathematics learning and attitudes. In World Conference on Educational Multimedia, Hypermedia and Telecommunications, volume 2008, pages 447-452, 2008.

[32] Gwen Nugent, Brad Barker, Neal Grandgenett, and Vaicheslav Adamchuk. The use of digital manipulatives in k-12: Robotics, gps/gis and programming. In Frontiers in Education Conference, 2009. FIE'09. 39th IEEE, pages 1-6. IEEE, 2009.

[33] Shakir Hussain, Jörgen Lindh, and Ghazi Shukur. The effect of lego training on pupils' school performance in mathematics, problem solving ability and attitude: Swedish data. Educational Technology \& Society, 9(3):182-194, 2006.

[34] Jörgen Lindh and Thomas Holgersson. Does lego training stimulate pupils ability to solve logical problems? Computers \& education, 49(4):1097-1111, 2007.

[35] Rubén Mitnik, Matías Recabarren, Miguel Nussbaum, and Alvaro Soto. Collaborative robotic instruction: A graph teaching experience. Computers \& Education, 53(2):330-342, 2009.

[36] Lego Group. Lego mindstorms ev3. http://www.lego.com/ en-us/mindstorms/products/31313-mindstorms-ev3. Accessed March 19, 2015.

[37] Vex Robotics. Vex iq super kit. http://www. vexrobotics. com/super-kit.html. Accessed March 19, 2015.

[38] Fischertechnik GmbH. Robotics txt discovery set. http: //www.fischertechnik.de/en/desktopdefault. aspx/tabid-21/39_read-307/usetemplate-2_column_ pano/. Accessed March 19, 2015.

[39] Lego Group. Lego mindstorms nxt 2.0. http://shop. lego. com/en-US/LEGO-MINDSTORMS-NXT-2-0-8547. Accessed March 19, 2015.

[40] Omar Mubin, Catherine J Stevens, Suleman Shahid, Abdullah Al Mahmud, and Jian-Jie Dong. A review of the applicability of robots in education. Journal of Technology in Education and Learning, 1, 2013.

[41] Junun Robotics. Mark iii. http://www. junun.org/MarkIII/ Info.jsp?item=1. Accessed March 19, 2015.

[42] Pololu Corporation. Pololu 3-pi. https://www.pololu.com/. Accessed March 19, 2015.

[43] Pololu Corporation. Pololu zumo 32u4 robot. https://www . pololu.com/. Accessed March 19, 2015.

[44] Arduino. Arduino starter kit. http://arduino.cc/en/Main/ Arduinostarterkit. Accessed March 19, 2015.

[45] Parallax Inc. Boe-bot robot. https://www.parallax.com/ product/boe-bot-robot. Accessed March 19, 2015.

[46] qfix Robotics. Roboterbausatz "omnibot". http://www. qfixshop.com/. Accessed March 19, 2015.

[47] Arrick Robotics. Arobot mobile robot for experimenters and educators. http://www.arrickrobotics.com/arobot/. Accessed March 19, 2015.

[48] Inc Images SI. Servobotics robotic arm model ra-02. http:// www.imagesco.com/robotics/arm.html. Accessed March 19, 2015.

[49] Lynxmotion. A15x robotic arm combo kit. http://www. lynxmotion.com/. Accessed March 19, 2015.

[50] Robotikits Direct. Robotic arm edge. http://www.owirobot. com/. Accessed March 19, 2015. 
[51] AREXX Engineering. Robot arm mini (ra2-hobby). http:// arexx.com/robot_arm/html/en/documentation.htm. Accessed March 19, 2015.

[52] MIT Computer Science and Artificial Intelligence Laboratory. The mit seg: An origami-inspired segway robot. https://sites. google.com/site/mitprintablerobots/. Accessed March $19,2015$.

[53] Michael Rubenstein, Christian Ahler, and Radhika Nagpal. Kilobot: A low cost scalable robot system for collective behaviors. In Robotics and Automation (ICRA), 2012 IEEE International Conference on, pages 3293-3298. IEEE, 2012.

[54] James McLurkin, Andrew J Lynch, Scott Rixner, Thomas W Barr, Alvin Chou, Kathleen Foster, and Siegfried Bilstein. A low-cost multi-robot system for research, teaching, and outreach. In Distributed Autonomous Robotic Systems, pages 597-609. Springer, 2013.

[55] Mário Saleiro, Bruna Carmo, Joao MF Rodrigues, and JM Hans du Buf. A low-cost classroom-oriented educational robotics system. In Social Robotics, pages 74-83. Springer, 2013.

[56] Juan Gonzalez-Gomez, Alberto Valero-Gomez, Andres Prieto-Moreno, and Mohamed Abderrahim. A new open source 3d-printable mobile robotic platform for education. In Advances in autonomous mini robots, pages 49-62. Springer, 2012.

[57] James Hilder, Rebecca Naylor, Artjoms Rizihs, Daniel Franks, and Jon Timmis. The pi swarm: A low-cost platform for swarm robotics research and education. In Advances in Autonomous Robotics Systems, pages 151-162. Springer, 2014.

[58] Zachary Dodds, Steven Santana, Brandt Erickson, Kamil Wnuk, Jessica Fisher, and Matt Livianu. Teaching robot localization with the evolution er1. Accessible Hands-on Artificial Intelligence and Robotics Education, pages 18-23, 2004.

[59] Francesco Mondada, Michael Bonani, Xavier Raemy, James Pugh, Christopher Cianci, Adam Klaptocz, Stephane Magnenat, JeanChristophe Zufferey, Dario Floreano, and Alcherio Martinoli. The e-puck, a robot designed for education in engineering. In Proceedings of the 9th conference on autonomous robot systems and competitions, volume 1, pages 59-65. IPCB: Instituto Politécnico de Castelo Branco, 2009.

[60] K-Team Corporation. K-team robots. http://www.k-team. com/. Accessed March 19, 2015.

[61] Fanny Riedo, Morgane Chevalier, Stéphane Magnenat, and Francesco Mondada. Thymio ii, a robot that grows wiser with children. In Advanced Robotics and its Social Impacts (ARSO), 2013 IEEE Workshop on, pages 187-193. IEEE, 2013.

[62] iRobot. irobot create 2 programmable robot. http://www. irobot.com/About-iRobot/STEM/Create-2.aspx. Accessed March 19, 2015.

[63] WowWee Group Limited. Wowwee rovio. http://www. wowwee. com/en/products/tech/telepresence/rovio/rovio. Accessed March 19, 2015.

[64] John Cummins, MQ Azhar, and Elizabeth Sklar. Using surveyor srv-1 robots to motivate cs1 students. In Proceedings of the AAAI 2008 Artificial Intelligence Education Colloquium, 2008.

[65] ADEPT MOBILEROBOTS LLC. Amigobot. http://www. mobilerobots.com/researchrobots/amigobot.aspx. Accessed March 19, 2015.

[66] Sarah Bergbreiter and Kristofer SJ Pister. Cotsbots: An off-the-shelf platform for distributed robotics. In Intelligent Robots and Systems, 2003.(IROS 2003). Proceedings. 2003 IEEE/RSJ International Conference on, volume 2, pages 1632-1637. IEEE, 2003.

[67] Gabriel T Sibley, Mohammad H Rahimi, and G Sukhatme. Robomote: A tiny mobile robot platform for large-scale ad-hoc sensor networks. In Robotics and Automation, 2002. Proceedings. ICRA'O2. IEEE International Conference on, volume 2, pages 1143-1148. IEEE, 2002.

[68] Gilles Caprari, Thomas Estier, and Roland Siegwart. Fascination of down scaling - alice the sugar cube robot. Journal of micromechatronics, 1(3):177-189, 2001.

[69] Kirstin Hagelskjaer Petersen, Radhika Nagpal, and Justin K Werfel. Termes: An autonomous robotic system for three-dimensional collective construction. 2011.

[70] M Brett McMickell, Bill Goodwine, and Luis Antonio Montestruque. Micabot: a robotic platform for large-scale distributed robotics. In ICRA, pages 1600-1605. Citeseer, 2003.

[71] Ali E Turgut, F Gokce, Hande Celikkanat, L Bayindir, and Erol Sahin. Kobot: A mobile robot designed specifically for swarm robotics research. Middle East Technical University, Ankara, Turkey, METUCENG-TR Tech. Rep, 5:2007, 2007.

[72] Mário Saleiro, Bruna Carmo, Joao M F Rodrigues, and J M Hans du Buf. A low-cost classroom-oriented educational robotics system. In Social Robotics, pages 74-83. Springer, 2013.

[73] Hannes Kaufmann. Construct3d: An augmented reality application for mathematics and geometry education. In Proceedings of the tenth ACM international conference on Multimedia, pages 656-657. ACM, 2002.

[74] PBS Kid. Cyberchase shape quest. http://pbskids.org/ apps/cyberchase-shape-quest.html. Accessed March 19, 2015.

[75] Gustav Taxén and Ambjörn Naeve. Cybermath: A shared virtual environment for mathematics exploration. Center for User Oriented IT Design, Royal Institute of Technology, Technical Report CID-129, Stockholm, Sweden, 2001.

[76] Matt Dunleavy, Chris Dede, and Rebecca Mitchell. Affordances and limitations of immersive participatory augmented reality simulations for teaching and learning. Journal of Science Education and Technology, 18(1):7-22, 2009.

[77] Hannes Kaufmann and Bernd Meyer. Simulating educational physical experiments in augmented reality. ACM, 2008.

[78] Chris Dede, Marilyn C Salzman, and R Bowen Loftin. Sciencespace: Virtual realities for learning complex and abstract scientific concepts. In Virtual Reality Annual International Symposium, 1996., Proceedings of the IEEE 1996, pages 246-252. IEEE, 1996.

[79] Nai Li, L Chang, Yuan Xun Gu, and HB-L Duh. Influences of ar-supported simulation on learning effectiveness in face-to-face collaborative learning for physics. In Advanced Learning Technologies (ICALT), 2011 11th IEEE International Conference on, pages 320322. IEEE, 2011.

[80] Tzung-Jin Lin, Henry Been-Lirn Duh, Nai Li, Hung-Yuan Wang, and Chin-Chung Tsai. An investigation of learners' collaborative knowledge construction performances and behavior patterns in an augmented reality simulation system. Computers \& Education, 68:314-321, 2013.

[81] Yelva C Larsen, Hagen Buchholz, Constantin Brosda, and Franz X Bogner. Evaluation of a portable and interactive augmented reality learning system by teachers and students. Augmented Reality in Education, 2011:47-56, 2011.

[82] María Blanca Ibáñez, Angela Di Serio, Diego Villarán, and Carlos Delgado Kloos. Experimenting with electromagnetism using augmented reality: Impact on flow student experience and educational effectiveness. Computers \& Education, 71:1-13, 2014.

[83] Wannisa Matcha and Dayang Rohaya Awang Rambli. Preliminary investigation on the use of augmented reality in collaborative learning. In Informatics Engineering and Information Science, pages 189-198. Springer, 2011.

[84] Su Cai, Feng-Kuang Chiang, and Xu Wang. Using the augmented reality $3 \mathrm{~d}$ technique for a convex imaging experiment in a physics course. International Journal of Engineering Education, 29(4):856865, 2013.

[85] Dimitris Alimisis. Educational robotics: Open questions and new challenges. Themes in Science and Technology Education, 6(1):pp-63, 2013.

[86] Sabine Kradolfer, Simon Dubois, Fanny Riedo, Francesco Mondada, and Farinaz Fassa. A sociological contribution to understanding the use of robots in schools: the thymio robot. In Social Robotics, pages 217-228. Springer, 2014

[87] M Shuttleworth. True experimental design. Retrieved [date of retrieval] from experiment resources http://www. experiment-resources. com/true-experimental-design. html, 2008.

[88] Jiwon Shin, Roland Yves Siegwart, and Stéphane Magnenat. Visual programming language for Thymio II robot. ETH-Zürich, 2014.

[89] Fanny Riedo, Philippe Rétornaz, Luc Bergeron, Nathalie Nyffeler, and Francesco Mondada. A two years informal learning experience using the thymio robot. In Advances in Autonomous Mini Robots, pages 37-48. Springer, 2012.

[90] Hsin-Kai Wu, Silvia Wen-Yu Lee, Hsin-Yi Chang, and Jyh-Chong Liang. Current status, opportunities and challenges of augmented reality in education. Computers \& Education, 62:41-49, 2013.

[91] Matt Dunleavy and Chris Dede. Augmented reality teaching and learning. In Handbook of research on educational communications and technology, pages 735-745. Springer, 2014. 